\title{
ICN/DTN for public safety in mobile networks
}

Schiller, Eryk ; Kalogeiton, Eirini ; Braun, Torsten ; Gomes, André ; Nikaein, Navid

\begin{abstract}
Long-Term Evolution (LTE) is a new communication standard developed by the Third Generation Partnership Project (3GPP). Currently, LTE is becoming a $4 \mathrm{G}$ reference architecture due to its widespread adoption among leading operators of mobile telecommunications. LTE is therefore foreseen as an important foundation for future $5 \mathrm{G}$ networks. In the shift towards $5 \mathrm{G}$, several open issues have to be worked out in LTE.
\end{abstract}

DOI: https://doi.org/10.1016/B978-1-78548-053-9.50011-1

Posted at the Zurich Open Repository and Archive, University of Zurich

ZORA URL: https://doi.org/10.5167/uzh-175059

Book Section

Accepted Version

Originally published at:

Schiller, Eryk; Kalogeiton, Eirini; Braun, Torsten; Gomes, André; Nikaein, Navid (2017). ICN/DTN for public safety in mobile networks. In: Daniel, Câmara; Nikaein, Navid. Wireless Public Safety Networks 3. London, UK: Elsevier, 231-247.

DOI: https://doi.org/10.1016/B978-1-78548-053-9.50011-1 
Chapter 11

\section{ICN/DTN for Public Safety in Mobile Networks}

\subsection{Introduction}

Long Term Evolution (LTE) is a new communication standard developed by the Third Generation Partnership Project (3GPP). Currently, LTE becomes a 4G reference architecture due to its wide-spread adoption among leading operators of mobile telephony. LTE is therefore foreseen as an important foundation of future $5 \mathrm{G}$ networks. In the shift towards 5G, several open issues have to be worked out in LTE. They emerge due to severe requirements put on the infrastructure of the future networks. First, mobile users will expect high capacity channels, in which capacity is measured in several Gbps. Second, new applications will be considered with high densities of connected devices. Third, $5 \mathrm{G}$ networks have to accommodate new types of connected devices such as household appliances, meters, connected cars. Fourth, direct device-to-device (D2D) communication has to be formulated for sharing information in a local context. In the broader view, network-based communication in the licensed can provide enhanced Quality of Service (QoS) for D2D scenarios. Fifth, extreme reliability (e.g., medical applications) and ultra-low latencies (e.g., VANET applications) have to be considered. Sixth, for Internet of Things (IoT) applications, communication of high energy efficiency is required. Finally, in current mobile networks, we are approaching Shannon's capacity limit. Therefore, an enhanced channel capacity shall be provided through the adoption of a new spectrum range. According to the aforementioned picture, $5 \mathrm{G}$ will be a holistic ecosystem providing connectivity

Chapter written by Eryk Schiller, Eirini Kalogeiton, Torsten Braun, André Gomes, Navid NIKAEIN. 
in a wide-range of application use-cases. It is therefore natural to seamlessly integrate Public Safety (PS) applications with 5G using LTE as a starting point.

LTE has been selected by National Public Safety Telecommunications Council (NPSTC) in the US as a basis for PS. Other regions of the world, such as the European Union, will most probably adapt LTE for PS as well. Currently existing PS systems such as Project 25 (P25), and Terrestrial Trunked Radio (TETRA) are already reliable in providing voice communication, however, new high bandwidth applications in PS can only be provided by LTE. Currently, LTE does not natively support PS as it was designed to support commercial cellular networking. It does not allow for a required level of reliability, security, and confidentiality. Moreover, the device-to-device communication is also not appropriately taken into account. Hence, a new research area emerges on an appropriate adaptation of LTE towards PS networking. 3GPP already addressed several of these issues in their studies such as device-to-device communication, Evolved Universal Terrestrial Terrestrial Radio Access Network (E-UTRAN), and Mission-Critical push-to-talk (MCPTT). 3GPP worked out specific properties of both the User Equipment (UE) and the evolved NodeB (the LTE base station eNB) that have to be taken into account to provision PS applications with respect to various availability levels of E-UTRAN. More specifically, 3GPP consider an isolated E-UTRAN scenario, in which an eNB operates with no access or limited connectivity to the LTE core (EPC). In such situations there is a need for rapid provisioning of the LTE network. The 3GPP does not study, however, how distributed disconnected (isolated) eNBs exchange information. This is left for vendors to implement their own proprietary solutions [FAV 16].

Delay Tolerant Networking (DTN) and Information Centric Networks (ICN) can provide an added value to LTE. Over the years, DTN has become an emerging pushbased paradigm for challenge networks. When a disaster occurs, communication has to be reestablished to ensure PS in areas, in which infrastructure is limited, power supplies might be damaged and/or the network is disconnected from its main core. DTN is an Internet architecture that overcomes technical difficulties that may exist in these challenge environments. In a disaster scenario, one must assume that nodes may be disconnected from their network and/or from each other. Hence, it is possible that information may not be immediately delivered to the destination due to a momentary lack of end-to-end connectivity.

The main advantage of DTN is that is enables communication in intermittent networks through its store-carry and forward mechanism. Every node in the network could store a message in its buffer when no connection is available. The node stores the message and can move to any direction, until a connection reappears. Then, the node forwards the message to other nodes so that the message can gradually approach the destination. In addition, another advantage of DTN includes the potential of working in heterogeneous networks (IP/non-IP) using the bundle protocol. Bundle protocol enables messages to be of variable sizes and enables the multi hop communication in 
order for a message to reach its final destination. This opportunistic model could change its routing decisions depending on the network topology. These two main advantages of DTN are exploited in PS to ensure that a message reaches rescue teams in disaster scenarios.

In addition, another concept addressing PS in disaster situations is ICN-based. ICN provides a pull strategy for content retrieval using content description for addressing purposes. It is radically different from current networks, which are using end-point identifiers to locate content. The store-carry and forward mechanism is an intrinsic property of ICN, since it does not rely on end to end communication, but instead communication is established based on the content of the exchanged messages and not on the locatiion of the host. It can therefore support intermittent connectivity in a catastrophic environment, i.e., scenarios, in which isolated nodes have to communicate with rescue teams.

To conclude, the aforementioned advantages of $5 \mathrm{G}$ and DTN/ICN make their combination a perfect candidate to ensure PS in catastrophic scenarios. The rest of this paper is structured as follows. In Section II, we present related work about MEC systems and DTN/ICN architectures. Section III presents the proposed MEC system architecture. In Section IV, we illustrate an example implementation of the MEC architecture, and finally we conclude in Section V.

\subsection{Related Work}

In Fig. 11.1, we depict a simplified schematic of the 4G Mobile Network Operator. It contains an evolved base stations (eNB), Mobility Management Entity (MME), Home Subscriber Server (HSS), Serving Gateway (SGW), and Packet Data Network Gateway (PGW). An eNB is a base station that provides a Radio Access Network (RAN) towards end-users operating User Equipments (UEs). The RAN is based upon Evolved Universal Terrestrial Radio Access (E-UTRA) using Orthogonal frequencydivision multiple access (OFDMA) on the downlink and Single-carrier FDMA (SCFDMA) on the uplink. An eNB communicates with the MME using the S1-AP protocol on the S1-MME interface and with the SGW using GTP-U protocol on the S1-U interface. An MME is a critical network function, which deals with the EPC control

plane. Its role is to manage sessions, authentication, paging, mobility, bearers, and roaming. It manages an eNB and an S-GW through the S1-MME and S11 interfaces respectively, and communicates with HSS through the S6 interface. An SGW is directly controlled by an MME. It is responsible for routing user plane packets between an eNB (S1-U) and a PGW (S5/S8 interface). It handles user handovers between neighboring eNBs. A PGW is a user plane component, which forwards packets between the LTE network and other packet networks. It is controlled through the S5/S8 interface by the SGW. Moreover, PGW is a mobility anchor for end-users. It performs charging and deep packet inspection (lawful interception) and manages Quality 


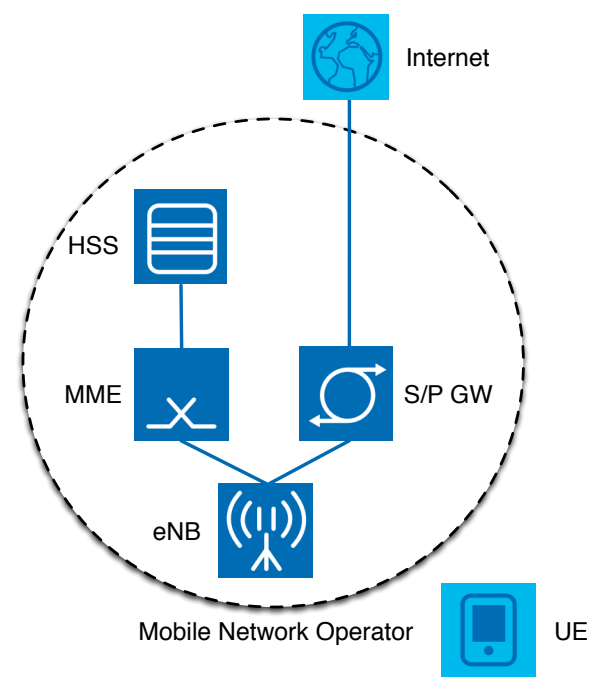

Figure 11.1. A simplified architecture of the LTE system.

of Service (QoS). HSS is responsible for maintaining the user service subscription information.

A typical E-UTRAN network can fail in various critical situations that can cause damages to various components of the $5 \mathrm{G}$ ecosystem. It results in a destroyed eNB, failed S1 interface disrupting the control and data plane, or damaged EPC. However, in PS communications, the network should guarantee a highly flexible and resilient operation in the case of various situations. Moreover, the PS network should adapt under various circumstances and mobility scenarios to provide a volatile infrastructure of high capacity.

To support local information processing and storage as well as improving robustness and providing low-delay communication to rescue and security teams, the concept of Mobile Edge Computing (MEC) seems a good candidate for provisioning critical systems at the network edge. The MEC concept is normally used to provide local computing and storage solutions at the eNB site. MEC is an extension of Mobile Networks of the future, i.e., $4 \mathrm{G}$ and 5G. Due to MEC, content, services, and applications can greatly benefit increased responsiveness of the network edge. MEC is also foreseen in $5 \mathrm{G}$ as an important technological enabler towards new genres of applications that intelligently combine geo location, network conditions, and radio information alltogether, to provide enriched services to end users. MEC is currently considered an important enabler of intelligent networking of the future and will widely spread in the $5 \mathrm{G}$ ecosystem. 
MEC Server

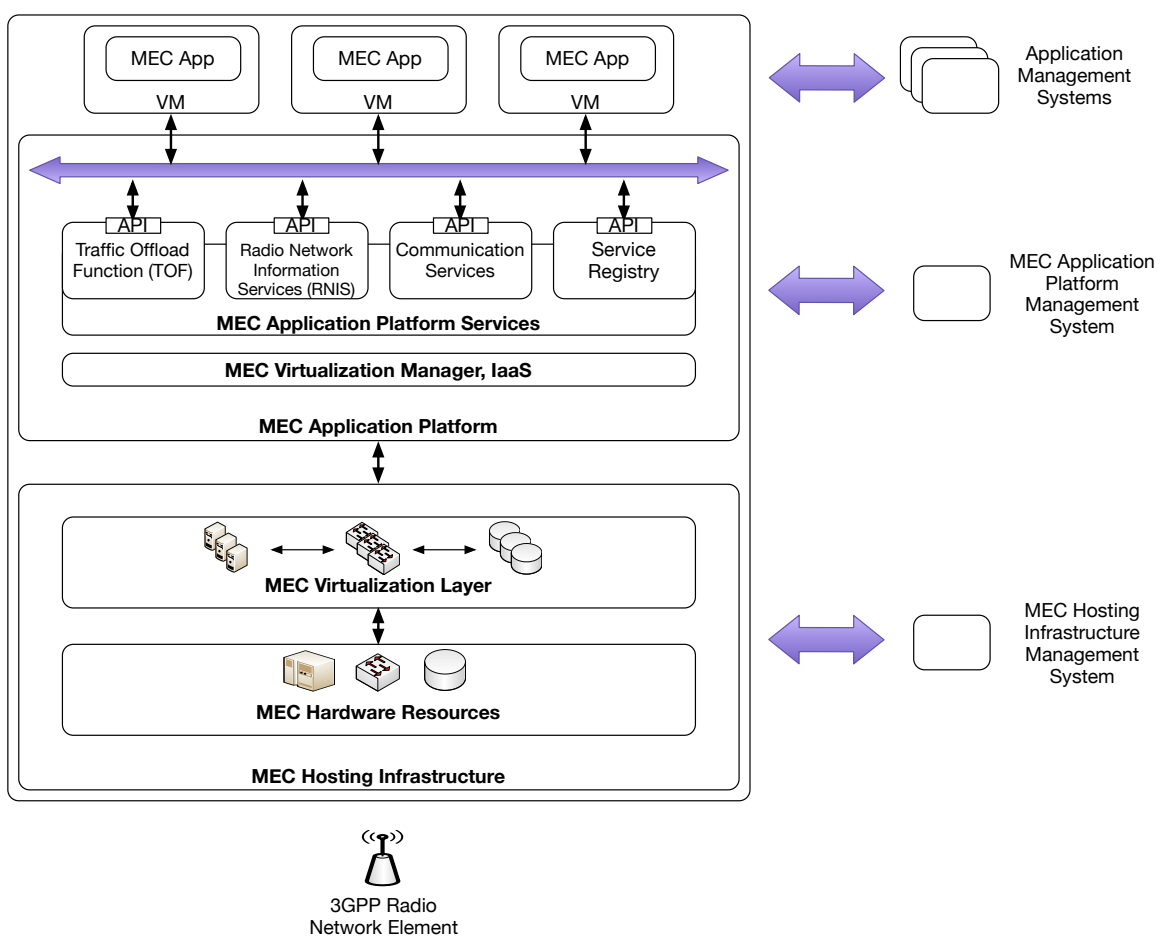

Figure 11.2. The architecture of the MEC system.

The architecture of a MEC system, which is in the ETSI white-paper [PAT 14], is illustrated in Figure 11.2. At the very bottom of the macro eNB site (c.f., Fig. 11.2), we illustrate a 3GPP-compliant radio interface. The MEC hosting infrastructure is provided at the bottom layer of the MEC server. It contains hardware resources (CPU, storage, and networking) as well as the MEC virtualization layer. Both hardware and virtualization techniques have to comply with the run-time requirements of MEC applications running on top. The upper MEC application platform consists of the MEC Virtualization Manager and MEC Application Platform Services. The virtualization manager is responsible for the life cycle of MEC Applications and communicates with MEC Virtualization Layer. The MEC application platform exposes an API to MEC Applications that is Traffic Offload Function (TOF), Radio Network Information Services (RNIS), Communication Services, and Service Registry. First, TOF redirects user traffic towards appropriate MEC applications based on various policies providing application chaining if requested. Second, the MEC application can be provided 
with radio channel information through RNIS. Third, the Service Registry holds information on currently maintained services. Finally, the Communication Services allow the MEC application to communicate all-together. MEC has been investigated in the FP7-MCN project [SOU 16]. In particular, it has been studied how MEC and ICN can complement each other, however, FP7-MCN focused on mobile networks with an operational core.

The utilization of DTN in disaster scenarios that ensures PS has been studied in literature. In [TRO 16], the authors propose a mapping system using a DTN environment in a disaster scenario. Specifically, they use civilians that act as sensor nodes through their mobile devices and collect Data. Nodes use DTN to transfer data reaching computing nodes that perform mapping of the affected area. Moreover, in [UCH 13] the combination of DTN with the Cognitive Wireless Network (CWN) for disaster networks is proposed. Furthermore, Fajardo et al. [FAJ 14] implemented a data collection method that uses people and their mobile phones as sensor nodes. People move inside an area of Interest to collect data, which is transmitted opportunistically. In addition, the authors propose message aggregation to reduce the message size and minimize the delay. Many DTN studies on a disaster scenario focus on the message transfer, i.e., the routing protocol that is used for message forwarding independently of the network situation (available resources, number of nodes). The authors in [VAH 00] propose the use of Epidemic routing to spread messages in the network. In particular, they develop a broadcast technique to exchange messages from a node to all its connections. In addition, in [LIN 03], the Probabilistic Routing Protocol using History of Encounters and Transitivity (PRoPHET) is proposed, where the authors use a learning phase to collect connectivity probabilities in the network. Specifically, each node maintains a possibility of meeting other nodes, and messages are exchanged based on that. Moreover, in [BUR 06], an extension of PRoPHET called "MaxProp" is proposed. It provides acknowledgments. The protocol prioritizes messages with a smaller number of intermediate hops. The protocol also keeps track of the previous message exchange to avoid duplicate transmissions. Spyropoulos et al. [SPY 05] introduced a new routing protocol Spray and Wait. This protocol operates two phases. First is spray, in which a certain number of messages is sent to other nodes. After that the wait phase is executed, in which nodes wait to observe whether the previously sent messages reached their destinations. Lastly in [DEM 07], a traditional Link State routing algorithm approach called "Delay Tolerant Link State Routing (DTLSR)" is suggested, where link state announcements are distributed through the network. For each message, the best path to the destination is selected, based on the link queue, the latency, and the bandwidth of the link.

Tyson et al. [TYS 14] study the utilization of ICN in disaster scenarios. The authors argue that ICN could improve connectivity resilience. This is due to the fact that in an ICN architecture, nodes can explore multiple interfaces at the same time. In addition, ICN does not have to maintain small connection timeouts as in classical networks. Finally, ICN requires no particular underlying network-layer, as it creates 
its own ad-hoc network. Moreover, deploying ICN in a network could improve QoS, as different requests could be treated differently. Furthermore, ICN supports the store, carry, and forward mechanism, as each node could be equipped with a cache, which is important in disaster scenarios, where connectivity may momentarily disappear. We therefore argue that the integration of DTN/ICN with LTE is an important research.

\subsection{System architecture}

In this subsection, we work out a mobile network architecture that provides ICN/DTN network services in the case, when a still functional eNB can provide a RAN towards end-users (UEs), but it does not have a valid connection to the network core. Note that when a fully functional eNB looses its connection to the core, it stops providing RAN. This situation can be a result of a failed S1 or EPC scenario (c.f., Fig. 11.3(a), Fig. 11.3(b)).

Strong protection mechanisms (such as independent power supplies, uninterruptible power supplies, etc.) can be used for MEC infrastructures similarly as previously used by legacy mobile telephony systems in the case of an eNB. Therefore, a MEC capable macro eNB site can independently operate for a long time after the critical event occurs, when the network core failed or the S1 interface malfunctioned. The macro enB site with MEC could therefore successfully provide communicating support to drones and rescue teams in the immediate vicinity even though the network core is not reachable. In this case, services can be provided in the failure case, according to local communication among attached nodes (in contrast to global communication when the remote core is available). We are therefore targeting the provisioning of disconnected E-UTRAN systems.

A macro MEC-enabled eNB is the main architectural element of the system. In the ordinary situation, when the network core is reachable, our MEC eNB site runs a software based Base-Band-Unit (BBU), which is a software part of an eNB that provides E-UTRAN and communicates with the operator network core to provide mobile access. The primary purpose of the MEC server is to run MEC applications that improve user quality of experience such as caching, online gaming, augmented reality, etc. Due to MEC, the base station can already actively cooperate in the DTN/ICN information dissemination by instantiating DTN/ICN based services as Virtual Network Functions (VNFs). The primary purpose of this work is to provide DTN/ICN in a disaster situation, when a bundle of a micro LTE core is provided to run RAN integrated with DTN/ICN.

Whenever a local Application Management Unit discovers that an eNB becomes disconnected from the core network, and that the eNB is not able to operate as an isolated eNB, it starts the recovery procedure to provision a new communication service. 


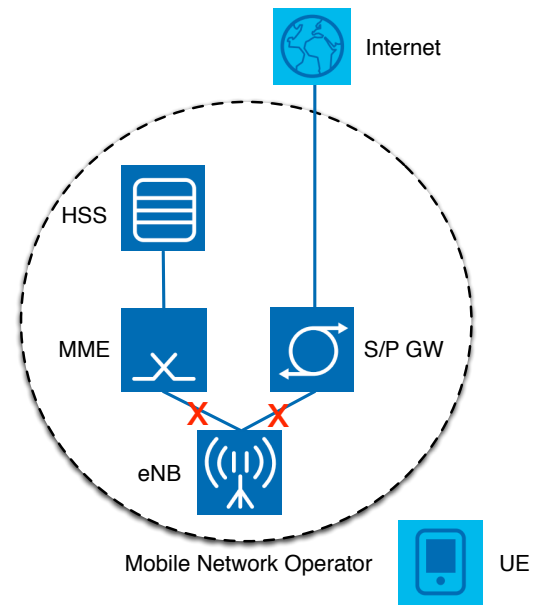

(a) Failed S1

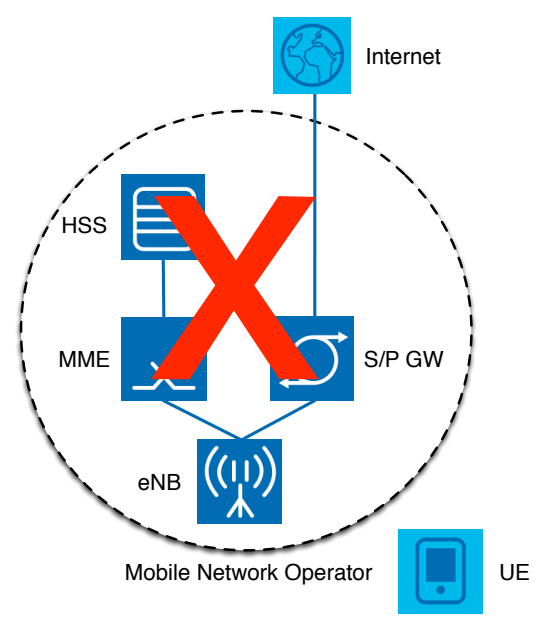

(b) Failed EPC

Figure 11.3. An operational eNB in the disconnected core scenario.

Such a service is deployed as a bundle of VNFs that defines the required network services, namely eNB, local S+P GW, MME, and HSS, and PS, and leverages the MEC platform when available to provide a fast network recovery and at the same time offer additional services (c.f., Figs. 11.4, 11.5). User information is maintained either by replicating the HSS database if possible, or by provisioning the known IMSI (range) 
MEC Server

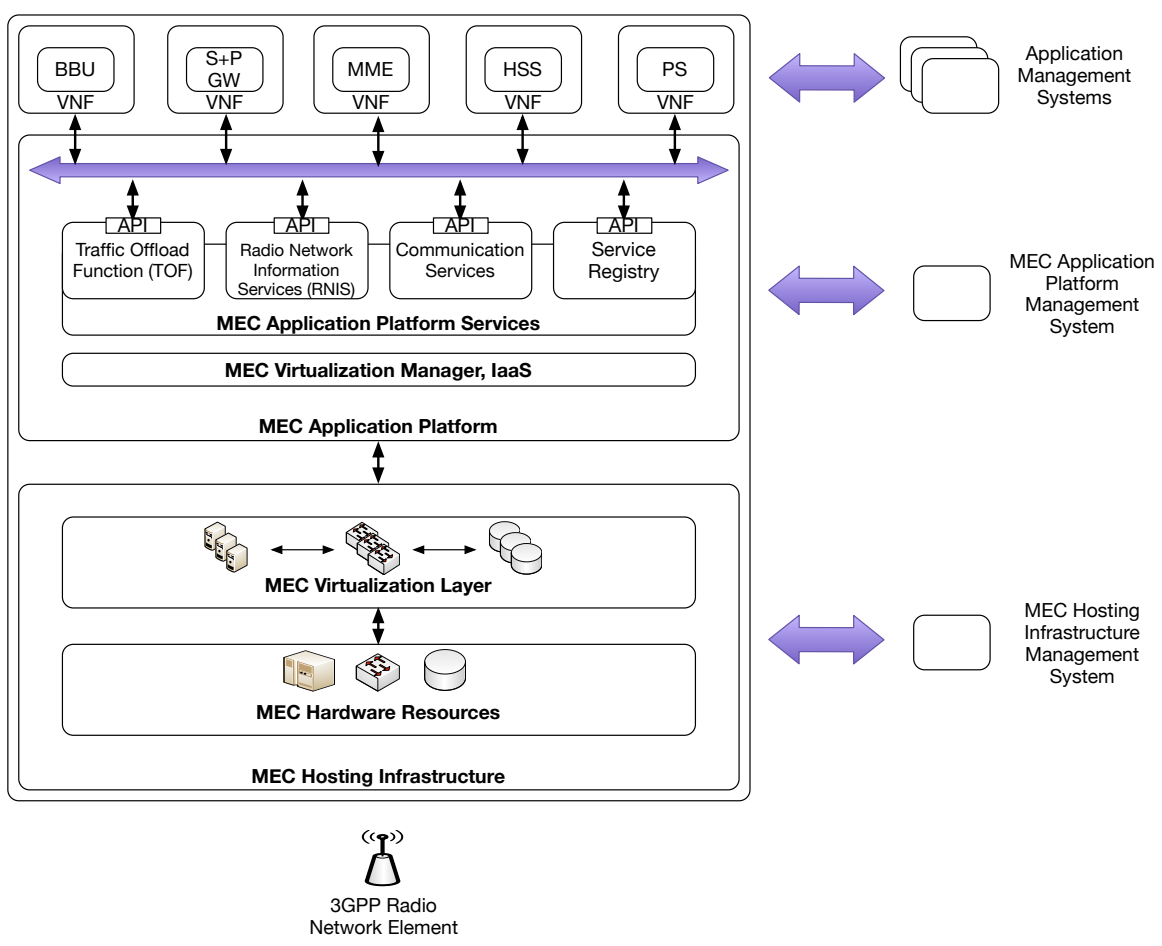

Figure 11.4. The architecture of the DTN/ICN PS Solution

without necessarily the operators key and sequence numbers. Note that the authentication procedure can also be relaxed so as to accept all the attach procedures.

All the VNF functions are instantiated on the the same edge cloud. The BBU has to be re-instantiated to acknowledge local copies of the MME, SPGW, HSS providing core network services. The MME, SPGW, and HSS are minimal services of a small footprint. They provide basic LTE functions and connect UEs attached with the macro eNB. Due to the basic core function, the UEs attached to the same eNB can communicate directly with the help of the PS VNF. The PS VNF is based upon DTN and/or ICN applications such as $\mathrm{CCNX}^{1}$, or DTN2 ${ }^{2}$. It is a communication end-point and a relay between other clients instantiated on UEs. The established setup allows end

1. http://blogs.parc.com/ccnx/ccnx-downloads/

2. https://sourceforge.net/projects/dtn/files/DTN2/ 


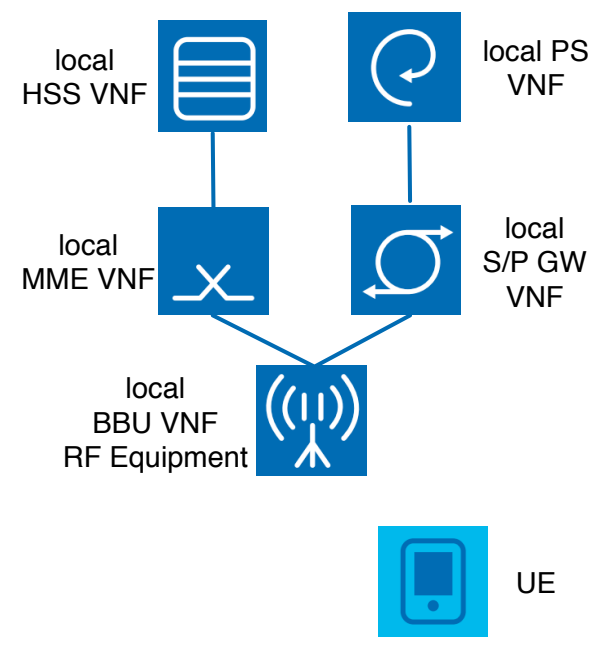

Figure 11.5. Service bundle for PS applications

users to attach to macro eNB (c.f., Fig. 11.6). The rescue teams can now freely attach to the open BTS instantiated and exchange data using DTN and/or ICN relay points. If a macro eNB shares a functional X2 interface with another nearby base station, the X2 can be used as an interface to share data among nearby cells. Otherwise, the cell-cell communication can be based upon DTN with data mules. DTN could enable a push communication between isolated eNBs, when a disaster has occurred. In particular, in disaster scenarios with no end-to-end communication, DTN can provide access to isolated areas through its main characteristics, which is delay tolerance. To this end we can consider this as a MANET, where users are equipped with devices (smartphones, tablets) and could potentially be used as data mules between the isolated nodes and the rescue teams, i.e. as shown in Fig. 11.6, in the case there is no functional interface between nearby cells, mobile users in an area could store data in their UE (i.e. mobile phones, tablets) and act as data mules transferring information from one cell to another.

ICN could perform a similar functionality. Although users are used as data mules, content is exchanged based on information in a pull manner. This is advantageous, since an isolated cell could not know the exact location of a functional cell and could just transmit Data based on the content, not on the location.

When connectivity to the regular core network restores, the service bundle is removed, and the local management entity establishes the original BBU again. 


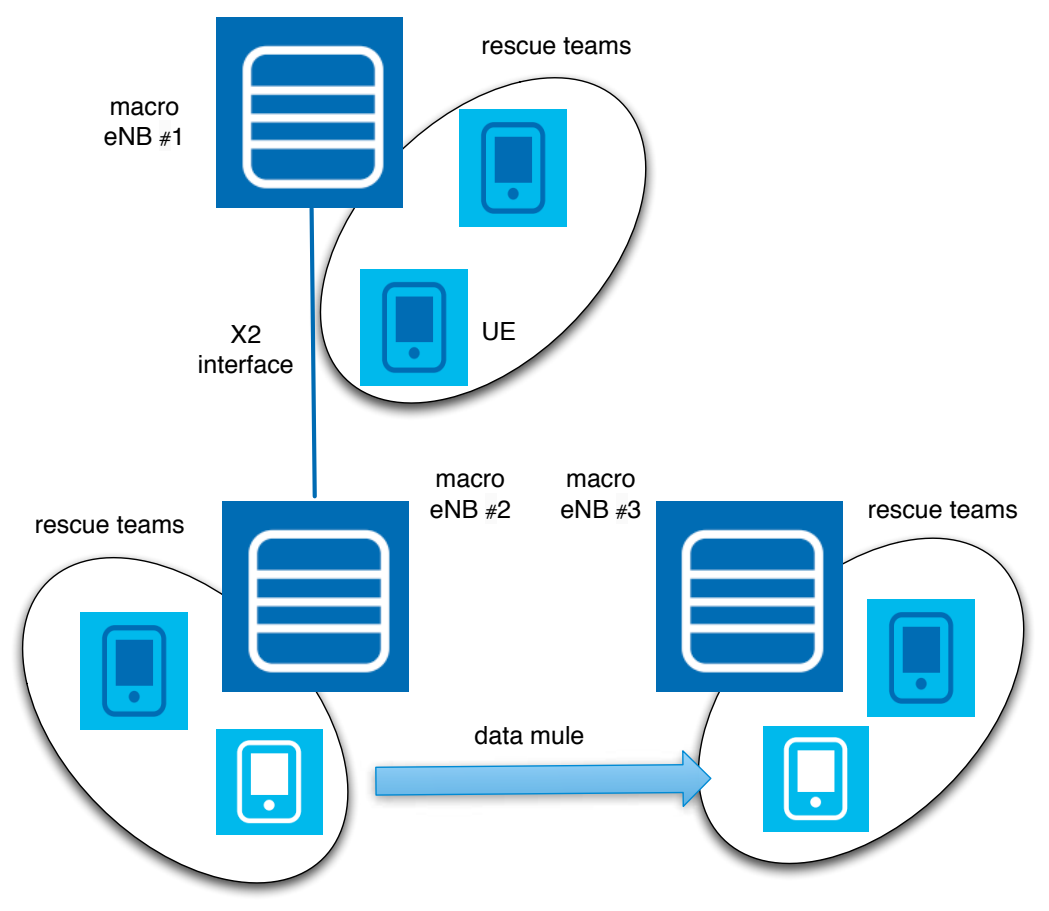

Figure 11.6. Service bundle functionality.

\subsection{Example Implementation}

\subsubsection{Juju VNF Manager}

The main building block of our system is Juju developed by Canonical. Juju is a domain neutral mechanism, which provides a generic VNF Manager (VNFM) that can be adopted to heterogeneous environments such as Infrastructure as a Service (IaaS) and Platform as a Service (PaaS) clouds. It natively supports service provisioning and scaling functions for scale-in/scale-out scenarios therefore dynamically handling workloads by properly adjusting resources to momentary situations.

Juju provisions on-demand various services provided as software. It spans large variety of applications such as databases (e.g., MySql), messaging systems (e.g., RabbitMQ), or monitoring infrastructures (e.g., Zabbix, Nagios) known from classical networks, but also more recently LTE network functions such as software-based BBUs, EPCs, HSS delivered by EURECOM as OpenAirInterface [NIK 14]. 


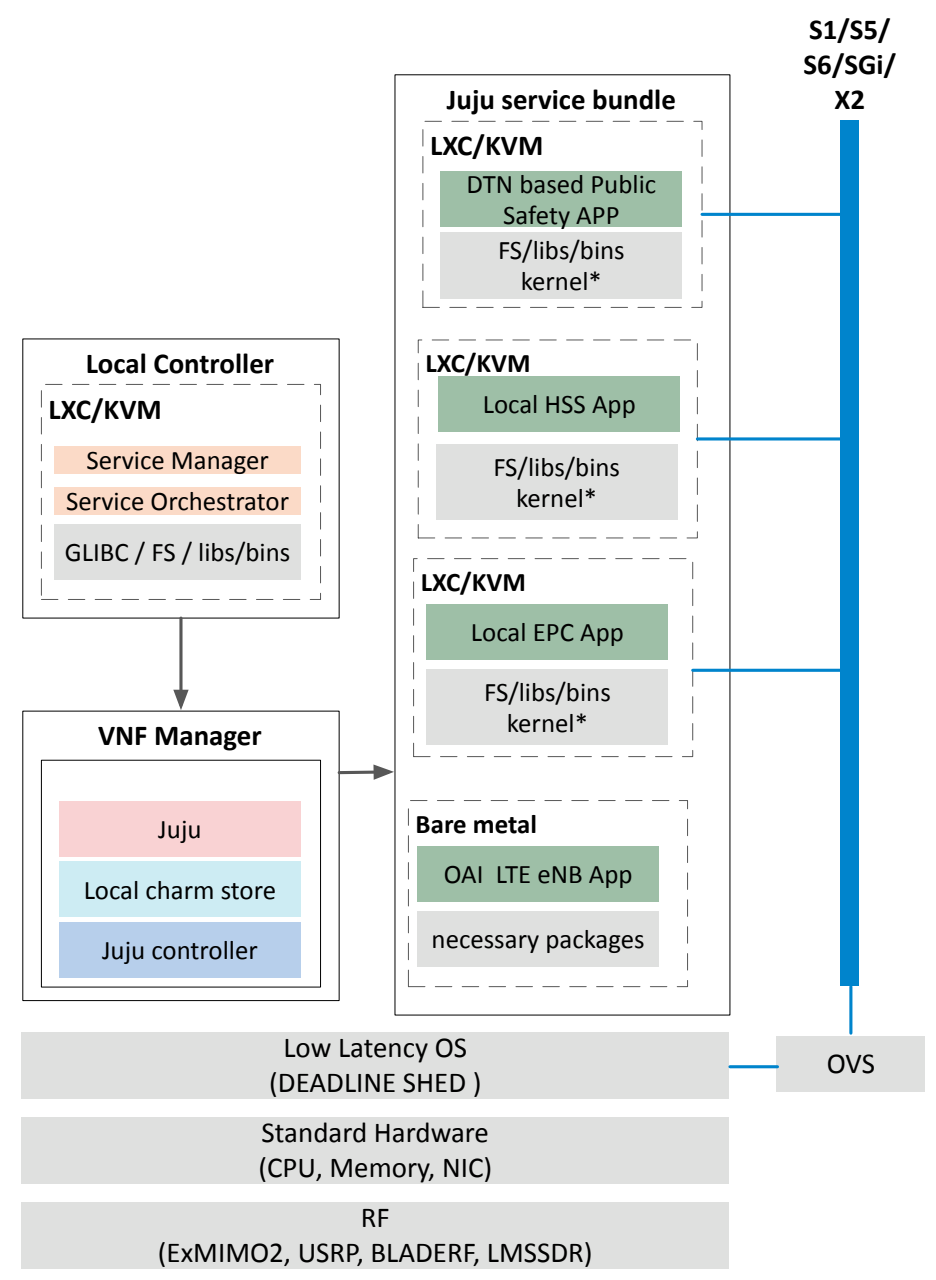

Figure 11.7. Software architecture.

Services are described by charms that are service manifests allowing for appropriate service configurations. Juju allows for "gluing" or "bundling" services all together by implementing logic allowing for automatic associations between services (i.e., service chaining). 
In the ETSI Management and Orchestration (MANO) ${ }^{3}$ architecture, Juju should be classified as a VNFM of extended capabilities, helping MANO vendors to implement advanced business logic in the service orchestration part to support an enhanced Quality of Service (QoS) through contracting appropriate Service Level Agreements (SLAs).

The hardware/software architecture of the system is presented in Fig. 11.7. At the bottom, we illustrate hardware resources (i.e., RF hardware such as ExpressMIMO2, Ettus USRP; computing infrastructure) used by the MEC. The following layer is the operating system of the host controlling the hardware, and providing network and computing virtualization tools (OpenvSwitch ${ }^{4}, \mathrm{KVM}^{5}$, XEN 6 , LXC 7 , etc.). Juju together with local charm store and juju controller play the role of the VNFM, which allows to spawn VNF bundles on the MEC infrastructure. The local controller instantiated through Juju is a VNF that monitors health of the network, and establishes the service bundle for PS applications when the eNB run with a disconnected core is detected through a telemetry service. The Juju service bundle for PS contains the whole LTE network stack, and a PS VNF connected with the help of the OVS switch providing a virtual network.

\subsubsection{OVS Virtual Switch}

The OVS is a crucial element of the designed setup. The main concern of the virtual switch is put on delay and throughput of the virtual infrastructure. To confirm network delay in virtual environment, we have created a laboratory setup using two computers directly connected with $1 \mathrm{GbE}-\mathrm{T}$ Ethernet cards in the University of Bern cloud laboratory. They both use Intel i7 CPU (host1: 4 cores, $3.6 \mathrm{GHz}, 32 \mathrm{~GB}$ RAM, Ubuntu 16.04, Kernel 4.4.0) and (host2: 2 cores, $3.4 \mathrm{GHz}, 16$ GB RAM, Ubuntu 16.04, Kernel 4.4.0) respectively. The faster machine, host1, is used to test the capacity of the virtual switch and various virtualization methods. We have tested the following configurations:

- Direct communication between two physical hosts with physical interfaces (A),

- Direct communication between two physical hosts; host1 has its physical interface configured as a port of an OVS switch. Two implementation of OVS are used: regular one (B), and Intel dpdk (C).

\footnotetext{
3. http://osm.etsi.org

4. http://www.openvswitch.org

5. http://www.linux-kvm.org

6. http://www.xenproject.org

7. http://linuxcontainers.org
} 


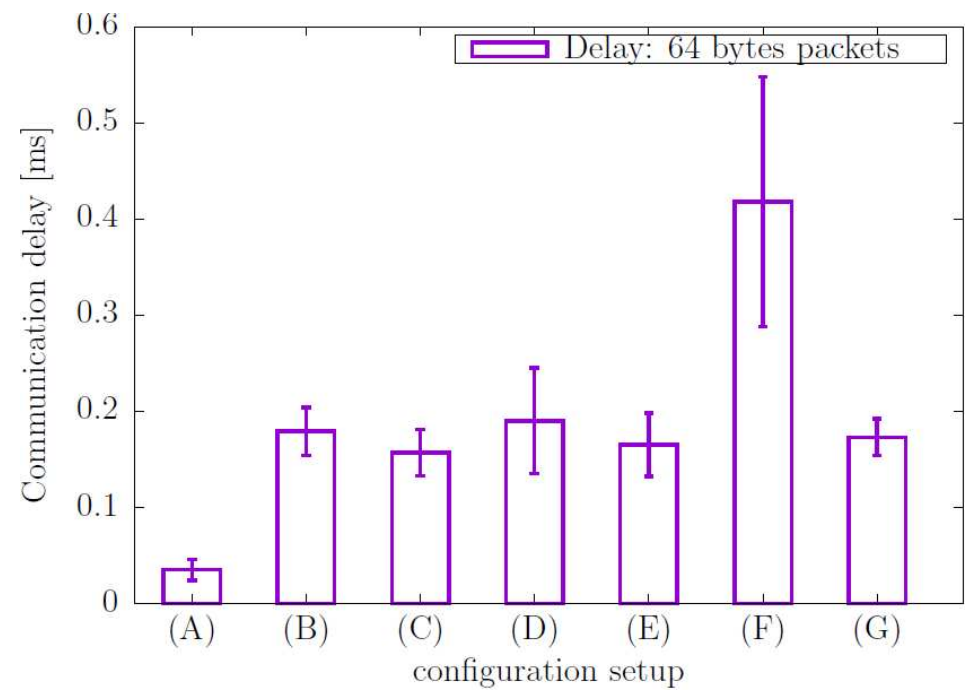

Figure 11.8. Delay of the OVS switch.

- Linux Container (LXC) instantiated on host1, communicates with hosts2. The physical interface of host1 is configured as a port of an OVS switch. Two implementations of OVS are used, again regular one (D), and dpdk (E).

- KVM instance on host1, communicates with host2. The physical interface of host 1 is configured as a port of an OVS switch. Two implementations of OVS are used, again regular one (F), and dpdk (G). In the regular mode, KVM uses tun/tap virtual interfaces configured as ports of the OVS. In dpdk mode, special dpdkvhostusers interfaces are used instead.

In modes (except dpdk), the OVS is able to saturate the $1 \mathrm{GbE}-\mathrm{T}$ link between host 1 and host 2 providing throughput of about $89 \mathrm{MB} / \mathrm{s} \pm 5 \mathrm{MB} / \mathrm{s}$. The dpdk mode displayed lower performance of around $60 \mathrm{MB} / \mathrm{s} \pm 1 \mathrm{MB} / \mathrm{s}$, however, more tests with different equipment are required to fully confirm this finding. The dpdk mode provides a dramatic improvement in the average communication delays (c.f., Fig. 11.8) especially in KVM. The delays were established by sending 64 bytes packets (ping) between communicating entities. The best performance of the virtual environment was established in mode (E), i.e., LXC with the dpdk OVS switch. It was then shortly followed by the KVM with dpdkvhostusers interfaces and dpdk OVS switch. We therefore see that the optimization of delay and throughput in OVS is getting closer to the performance of the physical environment 


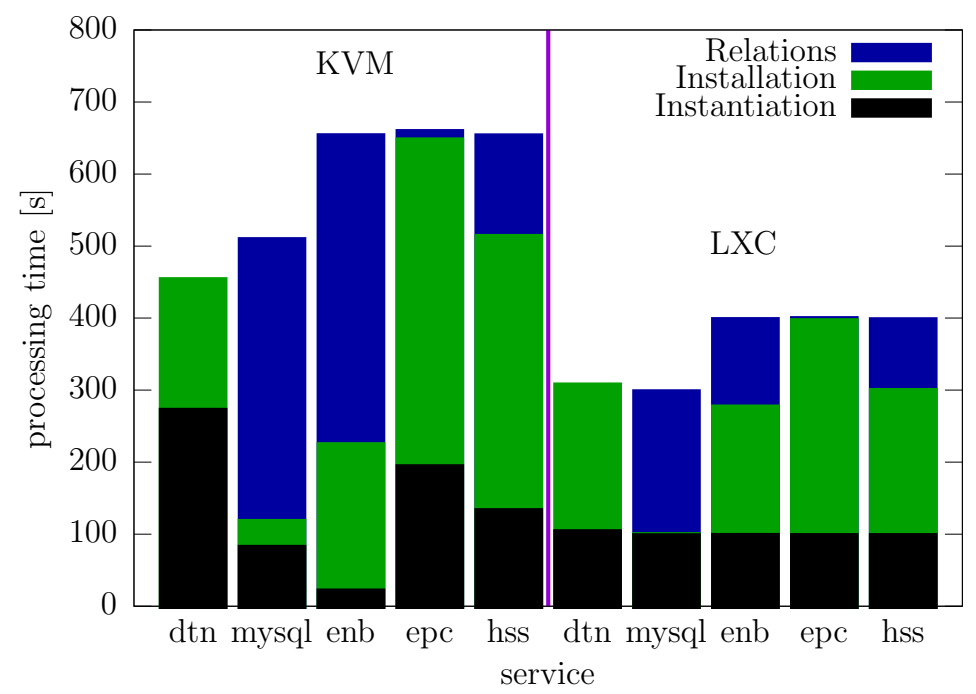

Figure 11.9. Provisioning time.

\subsubsection{PS bundle at the network edge}

Due to the LTE network stack containing a BBU, EPC, and HSS, the UE of the user can establish a connection with the network and connect to the PS application. In Fig. 11.9, we gather instantiation times for the PS service bundle (MySql is a supporting system for HSS). We tested two scenarios, when dtn (i.e., PS instance), mysql, epc, hss were instantiated on KVM and LXC respectively. In both scenarios, however, the eNB runs on the host as a bare metal service. This is to simplify the setup as in the bare metal mode, a pass-through between RF equipment and the container/VM is not necessary and the BBU can enjoy real-time capabilities of the host kernel. We use a single host machine with Intel $3.20 \mathrm{GHz}$ quad core CPU and 16 GB RAM. The services use 1 thread, 1 GB RAM; 1 thread 1 GB RAM; 4 threads 8 GB RAM; 1 thread, 2 GB RAM; 1 thread, 1 GB RAM for mysql, hss, enb, epc, and dtn resp.

Service provisioning contains three phases, i.e., instantiation (bare metal, LXC container, KVM VM), software installation, and relation establishment. In the relation establishment, the service installed earlier, mostly waits for its counterpart services to become ready. A reconfiguration is performed when another associated services become operational. For example, MySql waits for HSS to become ready. When it happens, both services bundle together and instantiation of MySql finishes. The enb, epc, hss, and dtn are not provided with binary packages, but compiled upon instantiation, therefore long installation times are expected. However, the complete PS stack requires around $650 \mathrm{~s}$ on KVM and $400 \mathrm{~s}$ on LXC to become fully provisioned. 
A UE can connect to the service (e.g., dtn in our scenario) and request data. We also noticed that on LXC, Juju creates containers in parallel, but deployment of VMs on KVM seems to be sequential.

\subsection{Conclusions}

We have developed an PS architecture for a macro eNB with MEC operating in a disconnected core scenario. MEC successfully establishes the whole LTE stack and provisions necessary PS services such as a DTN agent at the edge. The instantiation time is of about $650 \mathrm{~s}$ for KVM and $400 \mathrm{~s}$ on a typical commodity hardware, what is reasonable. Our solution can significantly improve the PS communication at the network edge. Future works include packaging the modules, thus improving instantiation time. We will also target other radio technologies such as WiFi. 


\section{Bibliography}

[BUR 06] Burgess J., Gallagher B., Jensen D., Levine B. N., "MaxProp: Routing for Vehicle-Based Disruption-Tolerant Networks.”, INFOCOM, vol. 6, p. 1-11, 2006.

[DEM 07] DEMMER M., FALL K., "DTLSR: delay tolerant routing for developing regions", Proceedings of the 2007 workshop on Networked systems for developing regions, ACM, Page 5, 2007.

[FAJ 14] Fajardo J. T. B., Yasumoto K., Shibata N., Sun W., Ito M., "Disaster information collection with opportunistic communication and message aggregation", Journal of information processing, vol. 22, num. 2, p. 106-117, Information Processing Society of Japan, 2014.

[FAV 16] Favraud R., Apostolaras A., Nikaein N., Korakis T., "Toward moving public safety networks", IEEE Communications Magazine, vol. 54, num. 3, p. 14-20, March 2016.

[LIN 03] Lindgren A., Doria A., SChelÉN O., "Probabilistic routing in intermittently connected networks", ACM SIGMOBILE mobile computing and communications review, vol. 7, num. 3, p. 19-20, ACM, 2003.

[NIK 14] Nikaein N., Knopp R., Kaltenberger F., Gauthier L., Bonnet C., NussBAUM D., GHadDab R., "Demo: OpenAirInterface: An Open LTE Network in a PC", Proceedings of the 20th Annual International Conference on Mobile Computing and Networking, MobiCom '14, New York, NY, USA, ACM, p. 305-308, 2014.

[Pat 14] Patel M., Naughton B., Chan C., Sprecher N., Abeta S., Neal A., "Mobile-Edge Computing - Introductory Technical White Paper", September 2014.

[SOU 16] Sousa B., Cordeiro L., Simoes P., Edmonds A., Ruiz S., Carella G. A., Corici M., Nikaein N., Gomes A. S., Schiller E., Braun T., Bohnert T. M., "Toward a Fully Cloudified Mobile Network Infrastructure", IEEE Transactions on Network and Service Management, vol. 13, num. 3, p. 547-563, Sept 2016.

[SPY 05] SPyropoulos T., Psounis K., RaghaVEndRa C. S., "Spray and wait: an efficient routing scheme for intermittently connected mobile networks", Proceedings of the 2005 ACM SIGCOMM workshop on Delay-tolerant networking, ACM, p. 252-259, 2005. 
[TRO 16] Trono E. M., Fujimoto M., Suwa H., Arakawa Y., Takai M., Yasumoto K., "Disaster area mapping using spatially-distributed computing nodes across a DTN", 2016 IEEE International Conference on Pervasive Computing and Communication Workshops (PerCom Workshops), IEEE, p. 1-6, 2016.

[TYS 14] Tyson G., Bodanese E., Bigham J., Mauthe A., "Beyond content delivery: Can icns help emergency scenarios?", IEEE Network, vol. 28, num. 3, p. 44-49, IEEE, 2014.

[UCH 13] Uchida N., Kawamura N., Williams N., Takahata K., Shibata Y., "Proposal of delay tolerant network with cognitive wireless network for disaster information network system", Advanced Information Networking and Applications Workshops (WAINA), 2013 27th International Conference on, IEEE, p. 249-254, 2013.

[VAH 00] VAhDAT A., Becker D. et al., "Epidemic routing for partially connected ad hoc networks", 2000. 